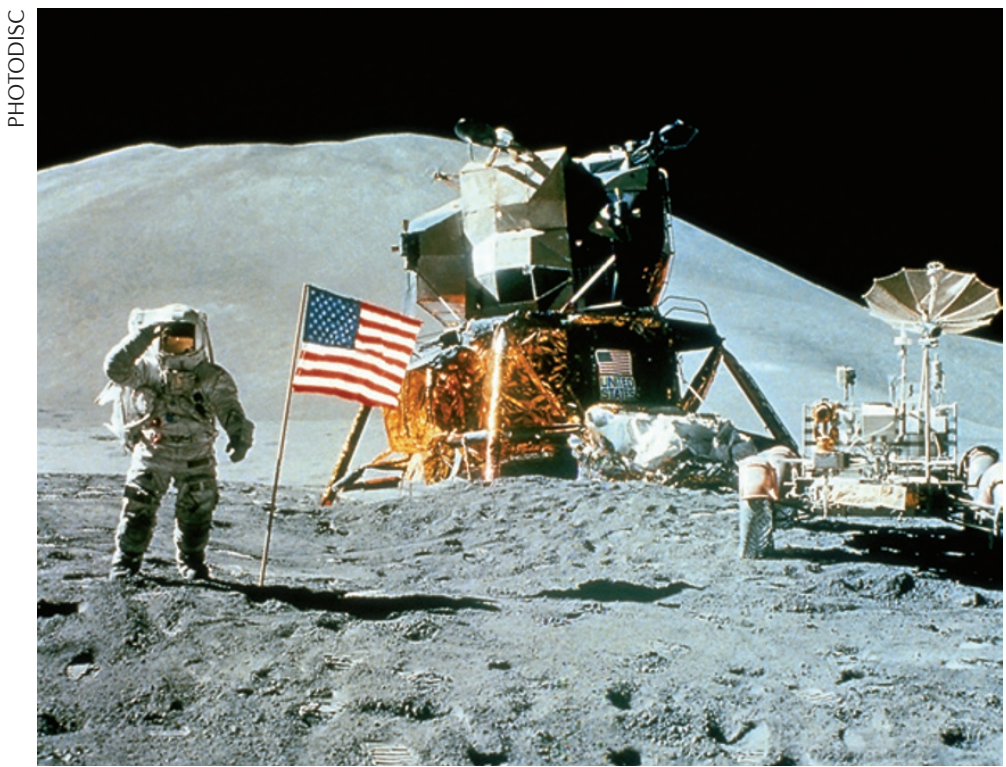

IMMUNE RESPONSES

\section{Platelets drive shuttle buggy}

Platelets are often overlooked by immunologists, but a new study by Verschoor et al. has shown that these blood components affect the course of systemic Listeria monocytogenes infection by promoting the transfer of blood-borne bacteria to splenic CD8 $a^{+}$dendritic cells (DCs). This function of platelets decreases the rate at which the host is able to clear the bacteria but, importantly, promotes the delivery of bacteria to CD8 $\alpha^{+}$DCs, enabling the development of adaptive immunity to the infection.

During systemic infection with L. monocytogenes, splenic CD8 $\alpha^{+}$ DCs have a crucial role in promoting antibacterial effector T cell responses, but they can also promote the spread of the infection by functioning as an early survival niche for L. monocytogenes. The authors set out to determine how bacteria in the blood are targeted to $\mathrm{CD}^{+} \mathrm{DCs}$ in the spleen and uncovered an important role for complement component $\mathrm{C} 3$ in this process. Compared with wild-type controls, C3-deficient mice had lower bacterial burdens in the spleen at days 1 and 3 following infection with L. monocytogenes, although both strains had overcome the infection by day 7. Using cobra venom factor to transiently deplete C3 in wild-type mice, the authors found that C3 depletion before, but not after, L. monocytogenes infection decreased bacterial loads. These findings are somewhat counterintuitive given the well-established roles of $\mathrm{C} 3$ in promoting the opsonization and lysis of microorganisms. This led the authors to propose that $\mathrm{C} 3$ might promote the transfer of L. monocytogenes to $\mathrm{CD} 8 \mathrm{a}^{+} \mathrm{DCs}$ in the spleen and therefore enhance bacterial survival at early time points after infection. But how does this occur?
Verschoor et al. analysed the appearance of blood-borne L. monocytogenes and found that almost all bacteria isolated from wild-type mice were associated with platelets. By contrast, this association was not seen when bacteria were isolated from C3-deficient mice. Depletion of platelets from wild-type mice led to lower bacterial burdens in CD8 $\alpha^{+}$DCs during $L$. monocytogenes infection. Furthermore, C3-mediated platelet binding seemed to target bacteria specifically to CD $8 \alpha^{+}$DCs. The binding of platelets to bacteria appeared to proceed through the alternative pathway of complement activation and required platelet expression of the glycoprotein GPIb. Additional experiments suggested that the associated platelets enhance targeting to $\mathrm{CD} 8 \alpha^{+}$ DCs by shielding bacteria from rapid degradation by macrophages. Notably, the authors found that platelets also associated with other Gram-positive strains of bacteria, revealing a broadly active shuttling mechanism for systemic bacteria.

Interestingly, failure of platelets to associate with $L$. monocytogenes inhibited the expansion of CD8 ${ }^{+}$ T cell populations with specificity for bacterial surface antigens. Therefore, platelet-mediated delivery of L. monocytogenes to $\mathrm{CD} 8 \mathrm{a}^{+} \mathrm{DCs}$ promotes the development of $\mathrm{CD}^{+} \mathrm{T}$ cells with specificity for bacterial antigens. The authors suggest that the C3and platelet-mediated shuttling mechanism could be manipulated for therapeutic purposes; for example, platelet association could be used to target antigens to CD $8 a^{+}$DCs.

Yvonne Bordon

ORIGINAL RESEARCH PAPER Verschoor, A. et al. A platelet-mediated system for shuttling bloodborne bacteria to $\mathrm{CD} 8 \alpha^{+}$dendritic cells depends on glycoprotein GPIb and complement C3. Nature Immunol. 30 Oct 2011 (doi: 10.1038/ni.2140) FURTHER READING Semple, J. et al. Platelets and the immune continuum. Nature Rev. Immunol. 11, 264-274 (2011) 